\title{
The Analysis of the Influence of Various Factors on the Development of Stress Corrosion Defects in the Main Gas Pipeline Walls in the Conditions of the European Part of the Russian Federation
}

\author{
A.V. Afanasyev, ${ }^{1}$ A. A. Mel'nikov, ${ }^{1}$ S. V. $\operatorname{Konovalov}^{\mathbb{D}},{ }^{1,2}$ and M. I. Vaskov $^{3}$ \\ ${ }^{1}$ Samara National Research University, 34 Moskovskoye Shosse, Samara 443086, Russia \\ ${ }^{2}$ Wuhan Textile University, 1 Fang Zhi Road, Wuhan 430073, China \\ 3"Gazprom Transgaz Samara" LLC, 106a Novo-Sadovaya Str., Samara 443086, Russia \\ Correspondence should be addressed to S. V. Konovalov; ksv@ssau.ru
}

Received 20 December 2017; Revised 17 April 2018; Accepted 2 May 2018; Published 3 June 2018

Academic Editor: Jerzy A. Szpunar

Copyright (C) 2018 A.V. Afanasyev et al. This is an open access article distributed under the Creative Commons Attribution License, which permits unrestricted use, distribution, and reproduction in any medium, provided the original work is properly cited.

\begin{abstract}
This paper considers the factors influencing the formation and development of stress corrosion defects detected during the inspection and overhaul of the main gas pipeline section. The surveyed gas pipeline is made of large diameter steel pipes made by controlled rolling, produced by various companies, with the predominance of pipes produced by the Khartsyzsk Pipe Plant (KhPP). The correlation between the geometric parameters of defects is described, which makes it possible to estimate the depth of cracks by external parameters. Mechanical tests by cyclic loading of samples containing cracks, based on the site operation data for the last 11 years, showed no crack growth in the absence of a corrosive medium. Micro-X-ray spectral analysis of metal and corrosion products showed no trace of the influence of hydrogen sulphide and nonmetallic inclusions (sulphides) on the development process of SCC. According to the results of the research, the process of development of stress corrosion on the main gas pipelines located in the European part of the Russian Federation is described. The organization operating the gas pipeline is recommended to take into consideration the results of this work during drawing up their repair plan.
\end{abstract}

\section{Introduction}

To date, more than a third (36\%) of accidents occur on the main gas pipelines (MG) of the Unified Gas Supply System (UGSS) belonging to "Gazprom» PJSC as a result of the development of stress corrosion defects or stress corrosion (hereinafter referred to as SCC). In the world practice of transporting natural gas through pipelines, this type of damage has the highest specific gravity among all other causes of accidents; therefore, during diagnostic examinations this type of defects is given the highest priority [1-3]. At the same time, along with the improvement of diagnostic tools, the number of newly detected CTC defects on UGS facilities is growing every year. If earlier, about 2,000 CWN defects were detected per year by methods of in-tube flaw detection (ILI) by magnetic shells; now with the use of electromagnetic-acoustic projectiles (EMA) this figure reaches 10,000 defects per year [4]. A large number of SCC defects are revealed by methods of nondestructive testing (NDT) in pits and during major overhauls. The absolute majority of defects detected by all inspection methods (almost 92\%) have a measured depth of less than $10 \%$ of the wall thickness $[5,6]$. According to experts, SCC crack of different depths was identified or is more likely to be detected in more than a million pipe sections during the next surveys [7-9]. This statement is consistent with the current trend to increase the number of small defects found.

The repair by replacing even a small part of these pipes will lead to a reduction in the total volume of UGSS pipeline overhaul due to the specific increase in the individual sections repair cost [10-12]. At the same time, the degree of defects' danger, the depth of which is less than $10-15 \%$ of the wall 


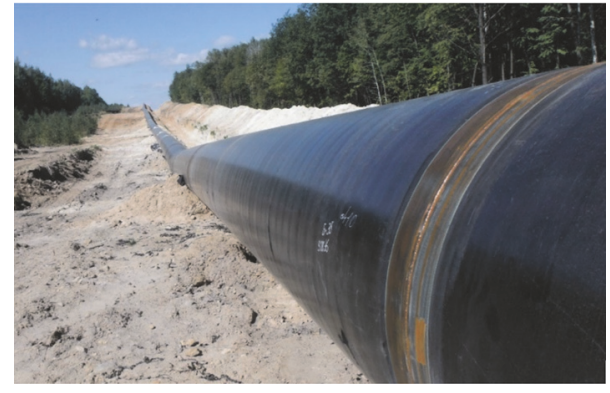

FIGURE 1: Investigated pipeline section.

thickness, under condition that access of corrosive medium to them is restricted, is determined by many researchers as insignificant [13-16]. At the present time, there are methods of repairing the pipelines polymer insulation, which do not allow the cracks to develop and threaten the reliability of pipelines for a long time [17, 18]. However, methods for an accurate assessment of the SCC cracks depth are not developed, and the factors that influence the distribution of SCC defects in extended sections are not always considered systematically during major overhauls.

Thus, the development of systematic methods of combating negative manifestations of stress corrosion in the form of accidents (identification, assessment, and targeted removal of only those defects that may lead to an accident in the foreseeable future with account of repair of insulation on other defects) is an urgent task.

The purpose of this study is to determine the factors that affect the distribution of SCC defects and the rate of their development during the main gas pipelines operation period. The second goal is to determine the parameters by which the depth of cracks can be estimated.

\section{Materials and Methods}

2.1. Field Studies on the Gas Pipeline Extended Section. For detailed consideration of operational factors and sampling, a section of main gas pipeline located in the eastern part of the European part of the Russian Federation with a length of 25 $\mathrm{km}$ was selected (Figure 1).

The selection criteria for the site were the presence of pipes of various types and the possibility of subsequent objective control of their technical condition. It was planned to evaluate the cyclicity over a long period of operation and compare the data of the ILI and the rejection results obtained during upcoming major overhaul (100\% NDT of the pipe surface). In addition, a comparison of the electrometric and physical profile of the section with a real distribution of SCC defects was carried out.

\subsection{Estimation of Cracks Depth by Their External Manifes-} tations. To create a mechanism for estimating the depth of SCC cracks using direct measurements of their geometric parameters, 15 samples were made from the rejected pipes, and 157 cracks were selected and described on these samples (Figure 2).
The surface of the samples was polished, the largest cracks in the colony were selected, and their length and width were measured. After that, the sample was cut and the depth of the cracks was directly measured on the cross section.

\subsection{Cyclic Tests with a Load Similar to the Operational Load.} To determine the effect of cyclic loading on the development of SCC defects under operating conditions with the excluded access of the corrosive medium to cracks, cyclic tests of 4 model samples were carried out under four-point loading conditions with accordance to the accepted industry method [19]. To justify the choice of cyclic test modes, the whole range of pressure fluctuations over a period of 11 years of operation was constructed based on the data from the operating modes log for selected pipeline section. The average value of the pressure $\left(\mathrm{P}_{\mathrm{av}}\right)$ was defined as $5.84 \mathrm{MPa}$ and the cycles with the greatest pressure deviations were identified. During the selected period, the pipeline section experienced 18 cycles of loading and unloading with the maximum deviation from the $\mathrm{P}_{\mathrm{av}}$ in the range of $17-35 \%$.

In the full spectrum of oscillations, a time interval (T) was determined, inside which the upper and lower halfperiods were analyzed. In these half-cycles, the cycles with the greatest amplitude deviations from $\mathrm{P}_{\mathrm{av}}$, equal to $2 \div 11 \%$ of the working pressure $\left(\mathrm{P}_{\mathrm{w}}\right)$, were identified. The parameters of the cyclic pressure change processes in each half-period $\left(\mathrm{P}_{\mathrm{cpc}}\right)$ were calculated (Figure 3 ). Based on the analysis of operating conditions of the investigated section of the main gas pipeline, it was found that the total number of pressure drop cycles within one year could be divided into two parts: 30 pulsation cycles with an amplitude in the range of $2 \div 11 \%$ of $\mathrm{P}_{\mathrm{w}}$ and 2 major cycles with an amplitude in the range of $17-35 \%$ of $\mathrm{P}_{\mathrm{w}}$.

As a result of the analysis the most conservative loading regime was chosen for the testing of model samples, which simulated the work of mail gas pipeline for 20 years of operation in real conditions. The load variation interval varied from 1.1 $\mathrm{MPa}$ to $7.4 \mathrm{MPa}$ (the maximum working pressure allowed on the pipeline section), which is characterized by the asymmetry coefficient of the cycle $(\mathrm{R}=0.15)$. The total number of cycles was calculated as the total number of all types of cycles affecting the gas pipeline for the year, multiplied by the planned interval of operation, equal to 20 years. Thus, the loading mode of the model samples was carried out in three stages with a change in the load every 640 cycles. The tests were carried out on a tensile machine using a four-point loading scheme (Figure 4).

To monitor the state of stress corrosion cracks, one control crack was selected on each sample, the photographic images of which were recorded with a metallographic microscope before the start of the tests, after the second stage, and at the end of the third stage of the test. The control was carried out relative to the initial state programmatically by measuring the number of pixels in the image along the line connecting the beginning and end of the crack.

2.4. Electron Microscopy. Cracks, cuts, and open corrosion cracks were studied by electron microscopy and micro-Xray spectral analysis. In the course of the study, micro-X-ray spectral analysis of the elemental composition of corrosion 


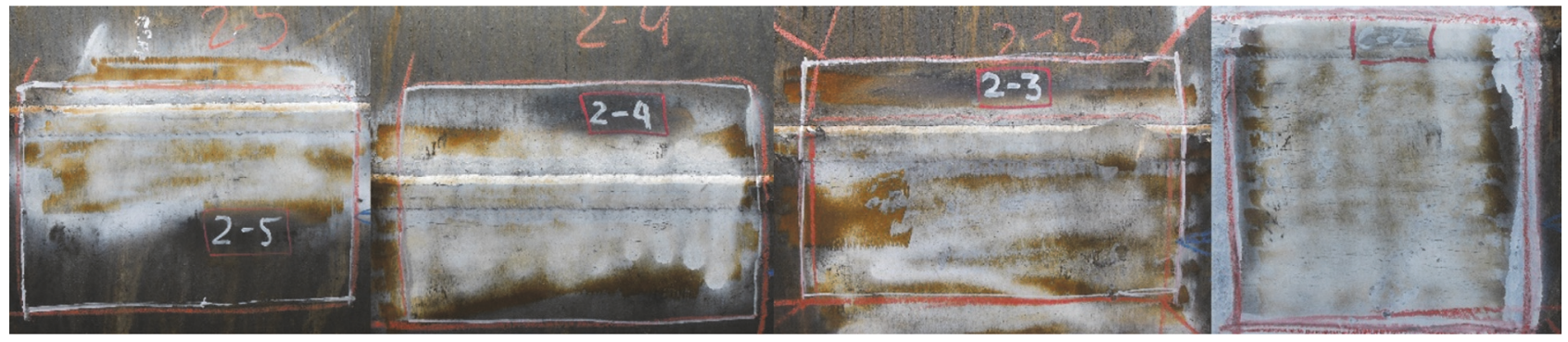

FIGURE 2: Samples with SCC cracks.


FIgURE 3: The upper and lower half-cycles of the oscillations in the chosen time interval (T).

products was carried out, and surface maps of the distribution of elements along the surface of destruction were made.

The fractographic and microscopic analysis of the crack surface was carried out on a TESCAN scanning electron microscope with VEGA software at magnifications up to 4000 times [19].

Spectral analysis to determine the quantitative chemical composition of the elements was carried out using the OXFORD INCAx-act energy dispersing attachment in accordance with ISO 15632: 2002.

Identification of the fracture behaviour by the type of surface relief was carried out in accordance with [20].

The general view of the TESCAN VEGA SBH Easy Probe scanning electron microscope and the OXFORD INCAx-act energy dispersive attachment is shown in Figure 5.

\section{Results and Discussion}

The density of the SCC defect distribution detected by the NDT during the overhaul was more than 97 times higher than expected by the results of the ILI. The average density of SCC defects on single-seam pipes (imported) is 50 times less than on double-seam pipes produced at Khartsyzsk Pipe Plant (KhPP). It was noted that stress corrosion damage localized mainly near the longitudinal weld. SCC defects of maximum depth (up to $36 \%$ of the wall thickness) were also found on the KhPP pipes.

The density of the distribution of SCC defects correlates with the number of KhPP pipes in the pipeline layout. At the same time, the noted characteristics of the external environment, as the value of the electrical resistance of the soil and the height differences along the section profile, do not significantly affect the distribution of defects. (Figure 6)

During the overhaul, more than $13.5 \mathrm{~km}$ of pipes were rejected on the site, more than $11.5 \mathrm{~km}$ (more than 85\%) of them due to SCC defects. In total, more than $58 \%$ of all the double-seam KhPP pipes inspected at the 25-kilometer section were rejected due to SCC defects. For single-seam pipes, including imported pipes, the rejection rate due to SCC was only $7 \%$.

The majority of defects detected during NDT (more than $92 \%$ ) have a depth of less than $10 \%$ of the wall thickness, which is below the detection threshold of magnetic in-tube flaw detectors. Therefore, undetected SCC defects can be present on other sections of the pipeline, and inspection with more sophisticated in-tube flaw detectors or NDT inspections in pits (for example, during the overhaul by reinsulation) will most likely reveal them.

During the research, it was established that there is a dependence between the main external parameters of SCC 



Every 50 cycles: Fixation of $h_{b}, h_{\text {end }}, F_{\text {up }}, F_{\text {low }}$

Every 640 cycles:

1. Photographing the ends of the notches on metallographic microscope

2. Fixing the test time

3. Raising the amount of load stress per 0.2 of sample yield strength

FIGURE 4: Cyclic testing of samples according to the four-point loading scheme.



Figure 5: General view of the TESCAN VEGA SBH Easy Probe scanning electron microscope.

cracks. The crack length-to-width ratio was $10: 1$ and the width-to-depth ratio was $0.06: 1$. Thus, at a depth of 1-1.5 mm, the opening width is $0.06 \mathrm{~mm}$; with a depth of $2-2.5 \mathrm{~mm}$, the opening width is $0.12 \mathrm{~mm}$ (Figures 7(a) and 7(b)).

Measurement of the crack opening width was carried out using a measuring magnifier with an accuracy of $\pm 0.01 \mathrm{~mm}$. In this case, the absolute limit error for the two measurements was $\pm 0.016 \mathrm{~mm}$ and the relative errors for each of the measurements were $\delta 1=28 \%($ section $\mathrm{A})$ and $\delta 2=24 \%$ (section $\mathrm{B})$, respectively.

After carrying out the cyclic tests, which were controlled by the metallographic method (Figures 8 and 9), it was found that, in the absence of a corrosive environment, the stress corrosion crack retained its original state after all the test stages. During the tests, no formation of new cracks-branches and other changes in the morphology of the crack apex was recorded. Thus, during the three stages of testing cycles simulating the operation of the real pipeline section (loadunload cycles), the development of existing stress corrosion cracks has not occurred (Figure 9).

Ten cracks were opened out by excessive force in order to study the resulting fracture. Analysis of the fracture surface at small magnifications showed that the upper part of the crack is completely filled with oxides. The soil brine constantly permeated and oxidized the internal surface of the crack during the operation life of the pipeline. The oxides formed also wedged and deformed the inner surface. Micro-X-ray spectral analysis of the elemental composition of corrosion products also showed that the cracks are completely filled with oxide. The distribution of various elements along the fracture surface is represented on the distribution maps of the elements (Figure 10).

As it can be seen from the distribution maps of the elements, there are no obvious phase boundaries. Corrosion 

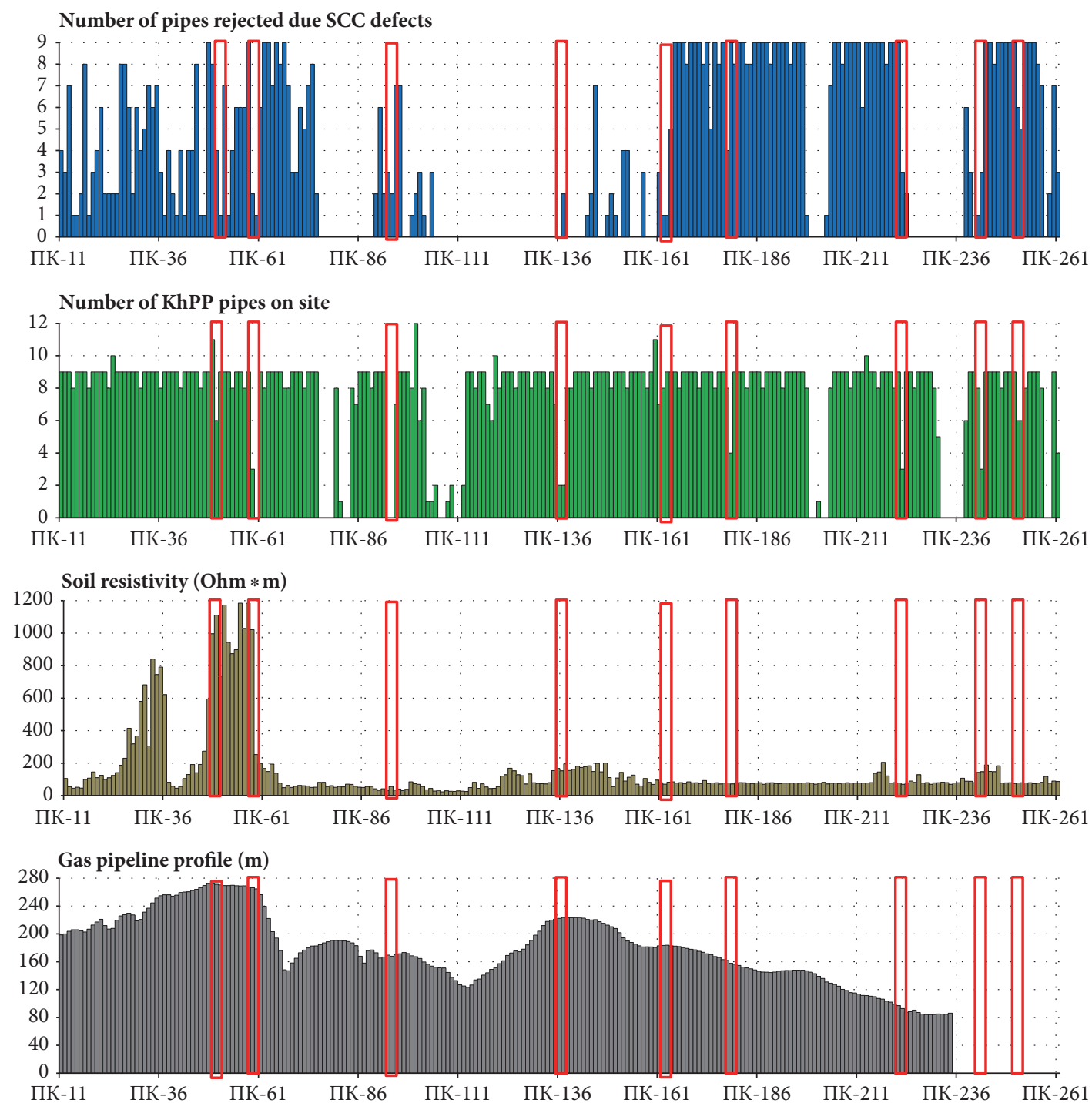

FIGURE 6: Number of pipes with SCC defects, number of KhPP pipes, soil characteristics, and section profile. The X-axis indicates the distance along the pipeline route. Pipeline sections with a small number of pipes produced by KhPP are marked in red.



(a)

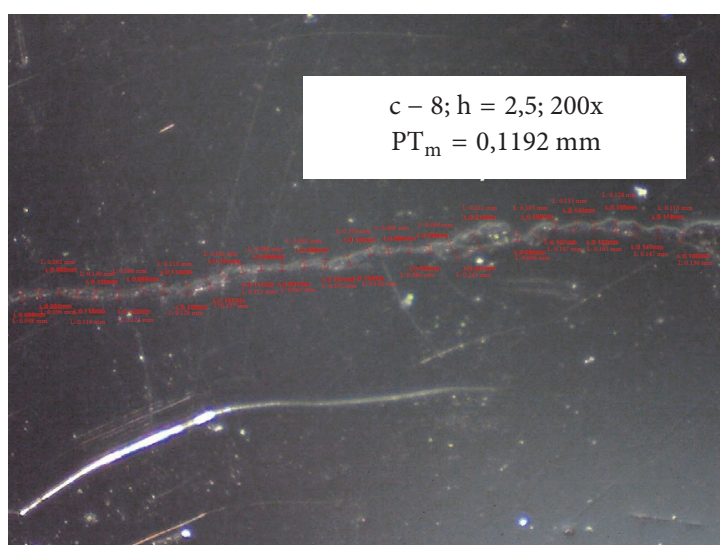

(b)

FIGURE 7: Microscopic examination of cracked sections. 


\section{Base metal}
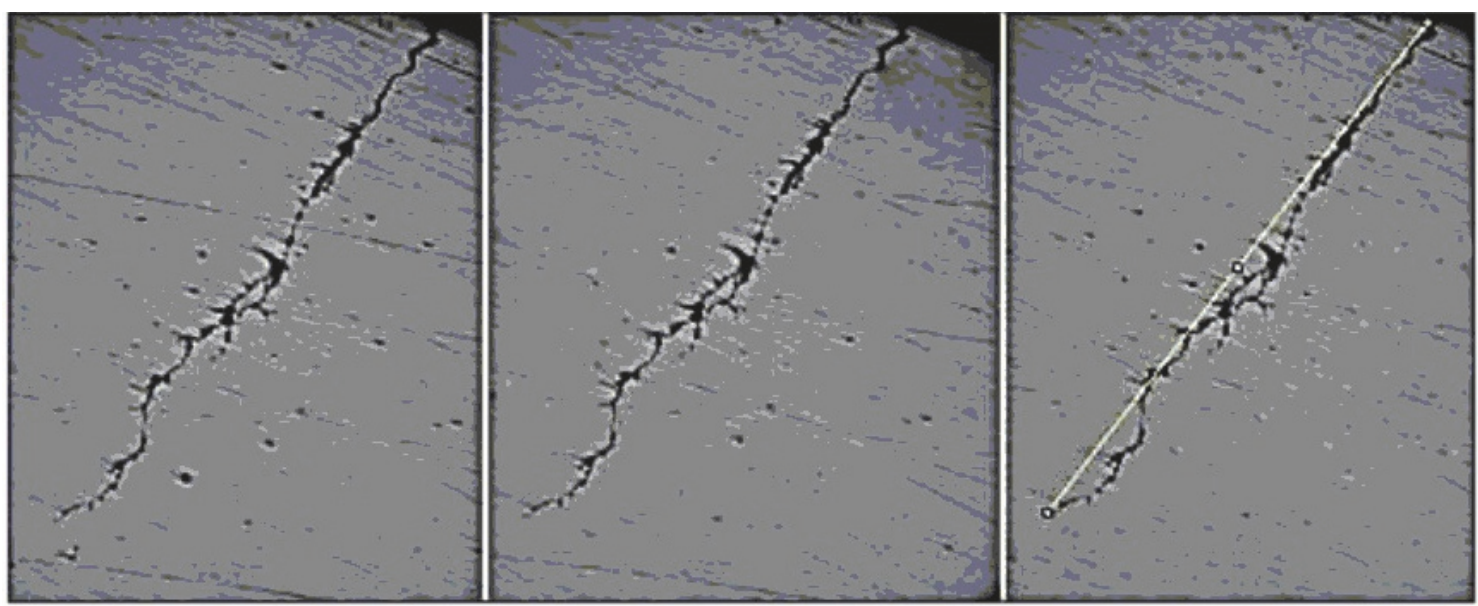

Results

$\begin{array}{cccc} & \text { Mean } & \text { Angle } & \text { Length } \\ 1 & 166.487 & -127.035 & 347.001 \\ 2 & 110.040 & -127.569 & 344.426 \\ 3 & 113.190 & -127.730 & 346.439\end{array}$

FIGURE 8: Results of crack depth stepwise measurement in the base metal of model sample.

weld metal
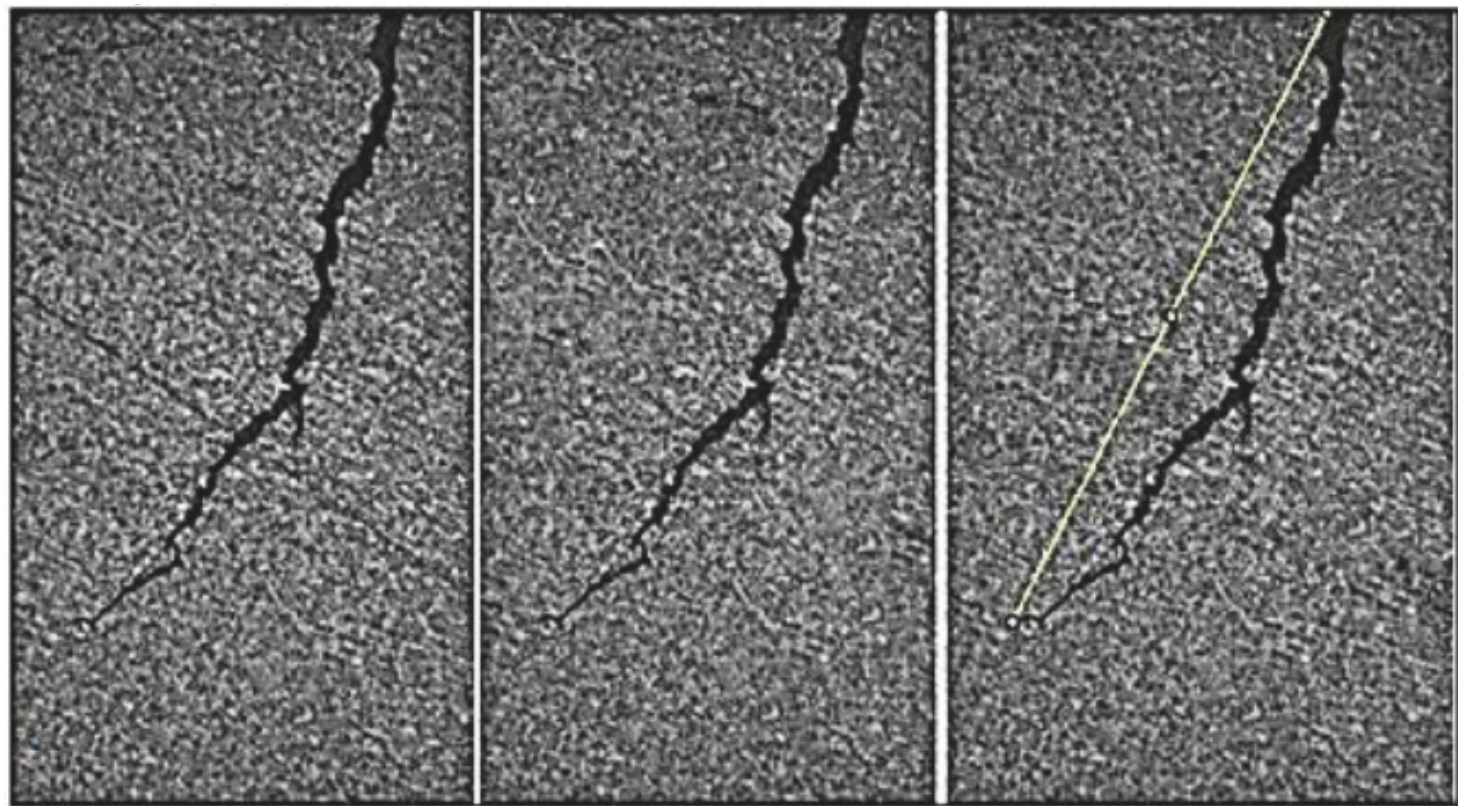

Results

Mean Angle Length

$106.151 \quad-117.282 \quad 286.021$

$104.792 \quad-117.196 \quad 284890$

$\begin{array}{lll}105.432 & -117.375 \quad 285118\end{array}$

FIGURE 9: Results of crack depth stepwise measurement in the model sample with longitudinal weld section. 




Electronic image

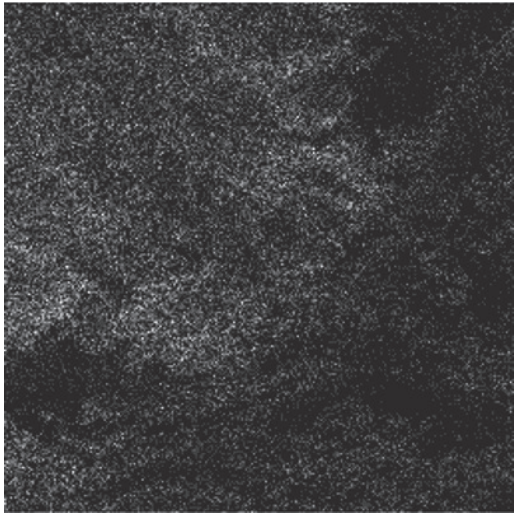

C Kal_2



O Ka1

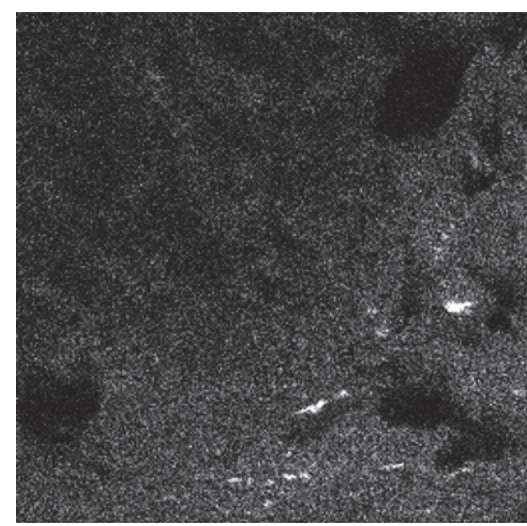

Mn Ka1



Fe Ka1

Figure 10: Elements distribution maps.



Electronic image

(a)



Mn Kal

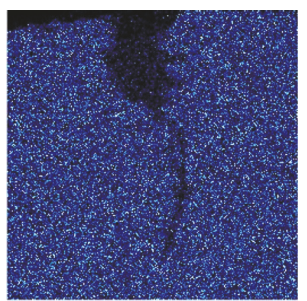

Fe Kal (b)



C Kal_2

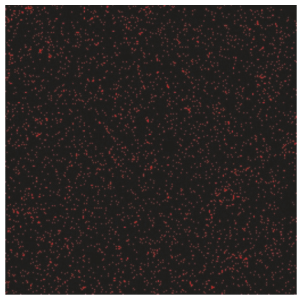

S Kal

(e)

FIGURE 11: Maps of elements distribution in a stress corrosion crack: (a) electronic image of the crack; (b) distribution of manganese; (c) distribution of iron; (d) distribution of carbon; (e) distribution of sulfur.

products are evenly distributed inside the crack. The images in the iron, oxygen, and carbon spectra are homogeneous and equally bright, indicating either a monophasic break in the oxide or even mixture of several phases. The expected composition of corrosion products is either a homogeneous $\mathrm{Fe}_{2} \mathrm{O}_{3}$ or a mixture of $\mathrm{Fe}_{2} \mathrm{O}_{3}$ and $\mathrm{FeCO}_{3}$.

To determine the effect of metallurgical impurities and additives on the development of cracks, the surface of the sections was also mapped in the spectra of sulfur and manganese. Due to limitations of the method, spectrometric data were not used to quantify the ratio of oxygen and carbon in corrosion products and in the base metal. During the mapping process, the main goal was to identify points with a local increased content of individual chemical elements corresponding to nonmetallic inclusions, their subsequent detailed analysis in case of revealing their influence on the process of material destruction, and subsequent detailed analysis of such points in case of revealing their influence on the process of material destruction.

The maps show an image of cracks in the samples in the spectra of iron, oxygen, carbon, sulfur, and manganese (Figure 11). An increased sulfur content in corrosion products was not observed, as evidenced by the different brightness of the elements spectrum image in the transition from the base 




Electronic image

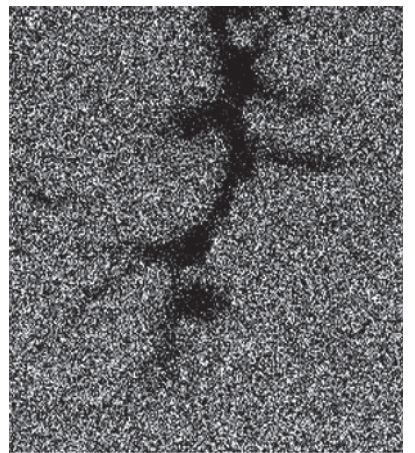

Fe Ka1

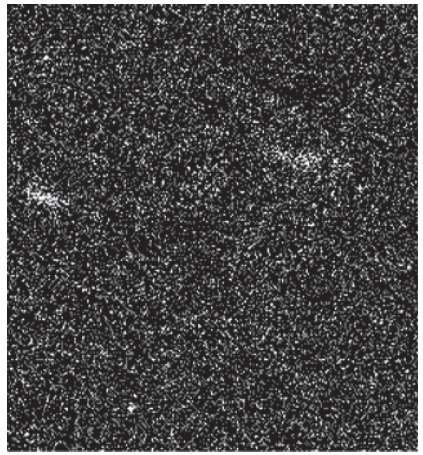

S Ka1

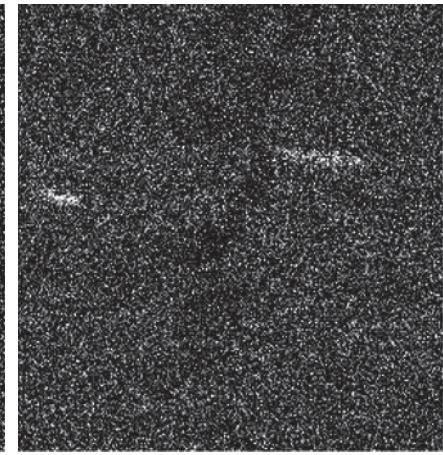

$\mathrm{Mn} \mathrm{Ka} 1$

FIGURE 12: Maps of elements distribution in a stress corrosion crack. Increased local sulfur content (manganese sulphide).

metal to the crack. A reduced content of iron and manganese and a high content of oxygen and carbon in the products of corrosion were noted (Figure 11).

The sulfur content in the corrosion products does not exceed the sulfur content in the base metal. Images in the spectra of manganese and sulfur are homogeneous and equally bright both in the cross section of the base metal and in the section of the crack. In some samples, a lower sulfur content can be noted with the exception of some local points (Figure 12).

Thus, the coincidence of the increased concentration of manganese and sulfur in some sections of the map indicates the presence of sulphide nonmetallic inclusion. As seen in the electronic image, these nonmetallic inclusions are not the sources of development of destruction. In the remaining volume of the crack, the sulfur content does not exceed the sulfur content of the metal, and the total contamination of the material with sulfur does not go beyond technical specifications.

Based on the data obtained as a result of the research, it is possible to assume a scenario for the development of SCC defects in the main gas pipeline.

During the construction of the pipeline, film insulation materials with on-site application were used, which during the period of operation lost their necessary technological properties. This is reflected in the fact that, during the operation of the pipeline, corrugations and pockets appeared in some places on the insulation coating. Corrosion-active soil electrolyte penetrated damaged insulation areas.

Later, these corrosion pits continued their growth and became concentrators of mechanical stresses in the pipeline wall. The source of these stresses were constant pressure pulsations from operating gas pumping units and gas temperature fluctuations due to the nonstationary operation of the gas compressor station.

The first microdeformations appeared, leading to the appearance of sharp cracks. Further, the cracks developed irregularly, under the influence of operational loads. In the development of the defect, a continuous process of corrosion was of decisive importance, stimulated by a constant inflow of heat along with the transported medium. The soil brine continued to penetrate into the cracks, wash out corrosion products, and deliver portions of the new electrolyte to the crack vertex.

Analysis of the effectiveness of various methods of nondestructive testing has shown that a colony of cracks can be identified by all available mass methods used in the oil and gas industry. However, in order to improve the efficiency of SCC defect detection, it is necessary to take into account and track factors that increase the predisposition of sites to this type of destruction.

The morphology of the detected defects corresponds to modern concepts of SCC defects. Defects are identified as cracks on the bottom of corrosive pits. They are grouped in colonies, oriented along the axis of the tube during their growth and branch along the section with the tendency to merge with one another.

The effect of sulfur and its compounds on the process of stress corrosion is not a significant factor, as there was no increased sulfur content in corrosion products. The phase composition of the corrosion products within the cracks is homogeneous across the crack cross section. The phase boundaries and the sections, which differ sharply in chemical composition, were not found.

Traces of the predominant effect of impurities and nonmetallic inclusions were not found. The structure of the metal is homogeneous and corresponds to the structure of rolled metal.

It was found that there is a consistent pattern between the width of the opening and the length of the crack. A simple statistical generalization showed that the length-towidth ratio could be described as 10:1 and the width-todepth ratio as 0.06:1. Since the majority of SCC cracks in the investigated samples are the same in origin, size, and morphology, it can be said that such a regularity exists in this particular case of SCC.

The appearance of cracks can be influenced by a variety of technological factors, laid down at various stages of the life cycle of the product. Such a factor can be the contamination of the pipe metal by harmful impurities and the presence of residual stresses in the pipe wall after and during manufacture at the stage of pipe production. In addition, these factors can include increased values of stresses in the pipeline wall, which arose due to flaws in construction design and installation 
work at the pipeline construction stage. This includes the nonprojected position of the pipe in the trench, unplanned soil movements, and welding stresses. The nonstationary operation mode of the pipeline also has a great influence, namely, the constant pulsation of the pressure and temperature of the pumped medium. Equally important is the corrosive activity of the ground in contact with the pipeline and the quality of the anticorrosive insulation at the stage of gas pipeline operation period.

Film insulation with on-site application does not provide long-term protection of the pipe body from SCC defects. After several years, due to soil movement and degradation of the coating, corrugations and pockets are formed in it, which collect soil moisture. Such conditions create a favorable environment for the development of corrosion defects. In the future, under the influence of these conditions and in the presence of an appropriate stress state, SCC defects can form. The insulation coatings used in newly constructed and repaired pipelines must provide long-term reliable protection of the pipe body against the corrosive effects of the environment in the operating conditions of main gas pipelines.

The main factor that can be detected and affects the distribution of stress corrosion, with other factors being equal, are the technological features of the pipe materials embedded in the product during its production stage. Presumably, this is the degree of local plastic deformation and the number of transitions during the formation of the pipe and sheet material and the thermal and high-speed welding of the longitudinal seam of two-seam pipes. To confirm this assumption, it is necessary to assess the residual stresses on the wall of the affected pipe and draw a map of them.

The presence of a corrosive medium is a prerequisite for the destruction of the pipeline wall due to the development of a SCC defect.

\section{Confirmations}

The presence of empirical dependence should be verified on a large statistical sample. Also, it is necessary to connect the external manifestations of SCC (the length of cracks and their opening) with the depth of stress corrosion damage. If such a relationship is confirmed in other cases of SCC, then it will be possible to describe the growth of a crack and the rate of its propagation in the general case with the sufficient accuracy. It will also be possible to build a mathematical model of the crack and calculate the residual life cycle of the pipeline using the finite element method.

Such an approach will make it safer to reuse the KhPP double-seam pipes during major overhaul, which is allowed only after a comprehensive evaluation of each pipe. In determining the degree of danger of each specific defect, a methodology for estimating the static strength of gas main pipeline with colonies of corrosion cracks is tested and used in "Gazprom transgaz Samara" LLC [21]. If, in addition to the nondestructive testing methods used in the survey, the external crack parameters are used and the calculation is performed using the largest depth obtained by different methods, then the assessment of the SCC crack danger will become more accurate.
The organization operating the gas pipeline is recommended to take into consideration the results of this work during drawing up their repair plan.

\section{Conflicts of Interest}

The authors declare that there are no conflicts of interest regarding the publication of this paper.

\section{References}

[1] S. V. Alimov, A. B. Arabej, and I. V. Ryaxovskix, The concept of diagnosis and repair of main gas pipelines in regions with a high predisposition to stress corrosion Gazovaya promyshlennost, vol. 724, 2015.

[2] Y. E. Cheng, Stress Corrosion of Pipeline, vol. 257, John \& Sons Publishing, Hoboken, NJ, USA, 2013.

[3] F. King, "Stress corrosion cracking of carbon steel used fuel containers in a Canadian deep geological repository in sedimentary rock," report No. NWMO TR-2010-21, Toronto, Canada, 2010.

[4] A. I. Mixajlov, Detection, identification and assessment of the depth of stress corrosion using combined magnetoacoustic in-tube flaw detectors// III Scientific and Practical Seminar "Increasing the Reliability of Main Gas Pipes Affected by Stress Corrosion Cracking", "Gazprom VNIIGAZ" LLC, Moscow, 2017.

[5] I. Ryakhovskikh, R. Bogdanov, T. Esiev, and A. Marshakov, "Stress corrosion cracking of pipeline steel in near-neutral $\mathrm{pH}$ environment," in Proceedings of Materials Science Technology, Pittsburgh, PA, USA, 2014.

[6] Y. A. Perlovich, O. A. Krymskaya, M. G. Isaenkova et al., "OP Conference Series: Materials Science and Engineering 10, Development, Production and Application," in Proceedings of the 10th International School-Conference on Materials for Extreme Environment: Development, Production and Application, MEEDPA 2015, p. 012009, 2016.

[7] A. I. Zaitsev, I. G. Rodionova, O. N. Baklanova, K. A. Udod, T. S. Esiev, and I. V. Ryakhovskikh, "Structural factors governing main gas pipeline steel stress corrosion cracking resistance," Metallurgist, vol. 57, no. 7-8, pp. 695-706, 2013.

[8] V. Linton, E. Gamboa, and M. Law, "Strategies for the repair of stress-corrosion cracked gas transmission pipelines: Assessment of the potential for fatigue failure of dormant stresscorrosion cracks due to cyclic pressure service," Journal of pipeline engineering, vol. 6, no. 4, pp. 207-217, 2007.

[9] U. Marewski, UKOPA/GP/009. Near neutral $p H$ and high $\mathrm{pH}$ stress corrosion cracking: industry good practice guide, U. Marewski and M. Steiner, Eds., UK onshore pipeline operators' association, Ambergate, Derbyshire, 2016.

[10] M. Gintten, "An integrated approach to the integrity management of stress corrosion cracking in pipelines: a case study," in Proceedings of the Proc. of Rio Pipeline Conference Exposions, M. Ginten, T. Penney, I. Richardson et al., Eds., 2013.

[11] A. B. Arabej, O. N. Melyoxin, I. V. Ryaxovskix et al., "Research into the possibility of long-term operation of pipes with low stress corrosion damage," Vesti gazovoj nauki, vol. 27, no. 3, pp. 4-11, 2016.

[12] R. V. Aginej, S. S. Gus'kov, V. V. Mussonov, R. A. Sadrtdinov, and V. A. Lapin, "Investigations of geometric parameters and peculiarities of the location of stress-corrosion damage on main gas pipelines," Vesti gazovoj nauki, vol. 27, no. 3, pp. 102-107, 2016. 
[13] R. Bogdanov, A. Marshakov, V. Ignatenko, I. Ryakhovskikh, and D. Bachurina, "Effect of hydrogen peroxide on crack growth rate in X70 pipeline steel in weak acid solution," Corrosion Engineering, Science and Technology, vol. 52, no. 4, pp. 294-301, 2017.

[14] W. Chen, R. Kania, R. Worthingham, and G. V. Boven, "Transgranular crack growth in the pipeline steels exposed to nearneutral $\mathrm{pH}$ soil aqueous solutions: The role of hydrogen," Acta Materialia, vol. 57, no. 20, pp. 6200-6214, 2009.

[15] X. Chen, Q. Yuan, B. Madigan, and W. Xue, "Long-term corrosion behavior of martensitic steel welds in static molten Pb-17Li alloy at 550॰C," Corrosion Science, vol. 96, pp. 178-185, 2015.

[16] X. Chen, Z. Shen, X. Chen, Y. Lei, and Q. Huang, "Corrosion behavior of CLAM steel weldment in flowing liquid Pb-17Li at 480 ॰c," Fusion Engineering and Design, vol. 86, no. 12, pp. 29432948, 2011.

[17] A. B. Arabey, O. N. Melekhin, O. V. Burutin et al., "Studying the Possibility of Long-Term Operation of Pipes with Insignificant SCC," 3R, no. 01-02, pp. 104-110, 2017.

[18] Y. Perlovich, O. Krymskaya, M. Isaenkova, N. Morozov, I. Ryakhovskikh, and T. Esiev, "Effect of layer-by-layer texture inhomogeneity on the stress corrosion of gas steel tubes," Materials Science Forum, vol. 879, pp. 1025-1030, 2017.

[19] C. J. B. Reed, Electron Probe Analysis and Raster Microscopy (Translation), Moscow, Texnosfera, 2008.

[20] RD 50-672-88. Methodical instructions. Calculations and strength tests. Classification of metals fracture types// Moscow, VNIIGAZ, 2010- $56 \mathrm{p}$.

[21] V. A. Subbotin, I. V. Scherbo, S. A. Kholodkov, and M. G. Giorbelidze, "Estimation of static strength of sections of the linear pipeline portion with a colony of corrosion fine cracks," Samara State Aerospace University Bulletin, vol. 47, no. 5, pp. 151157, 2015 (Russian). 


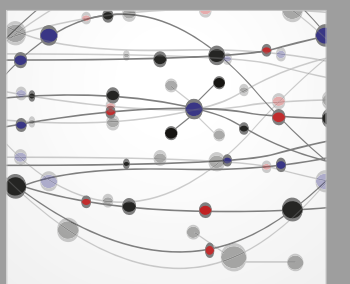

The Scientific World Journal
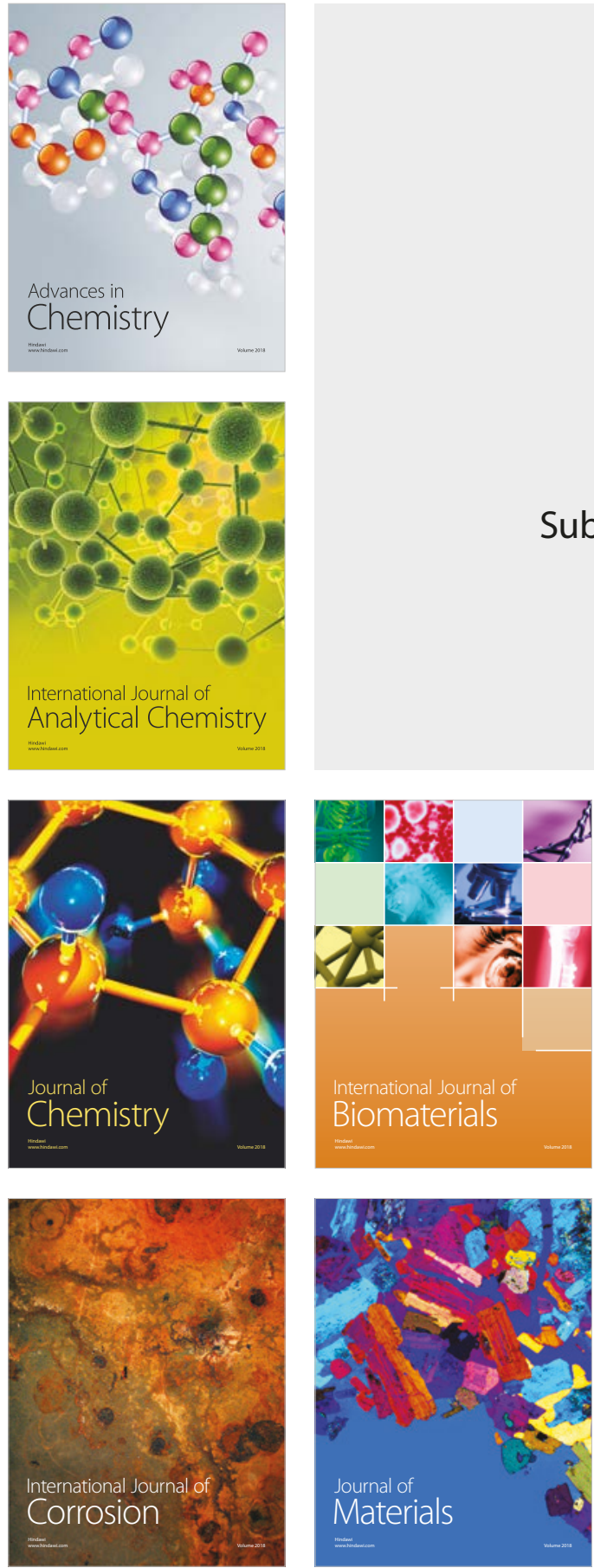

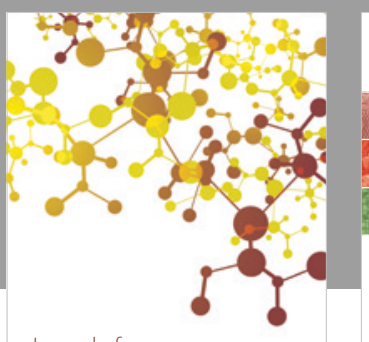

Journal of

Applied Chemistry
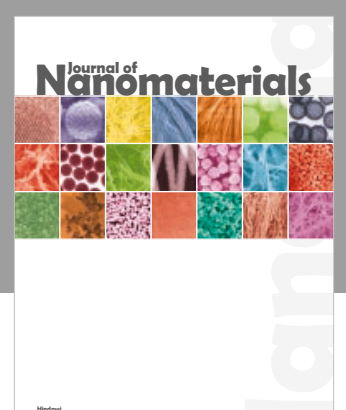

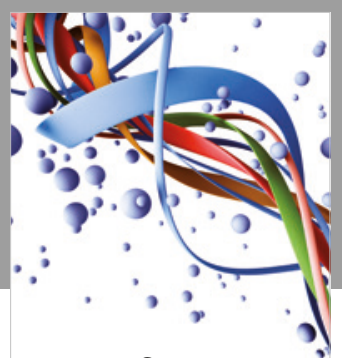

Scientifica



Polymer Science



Physical Chemistry
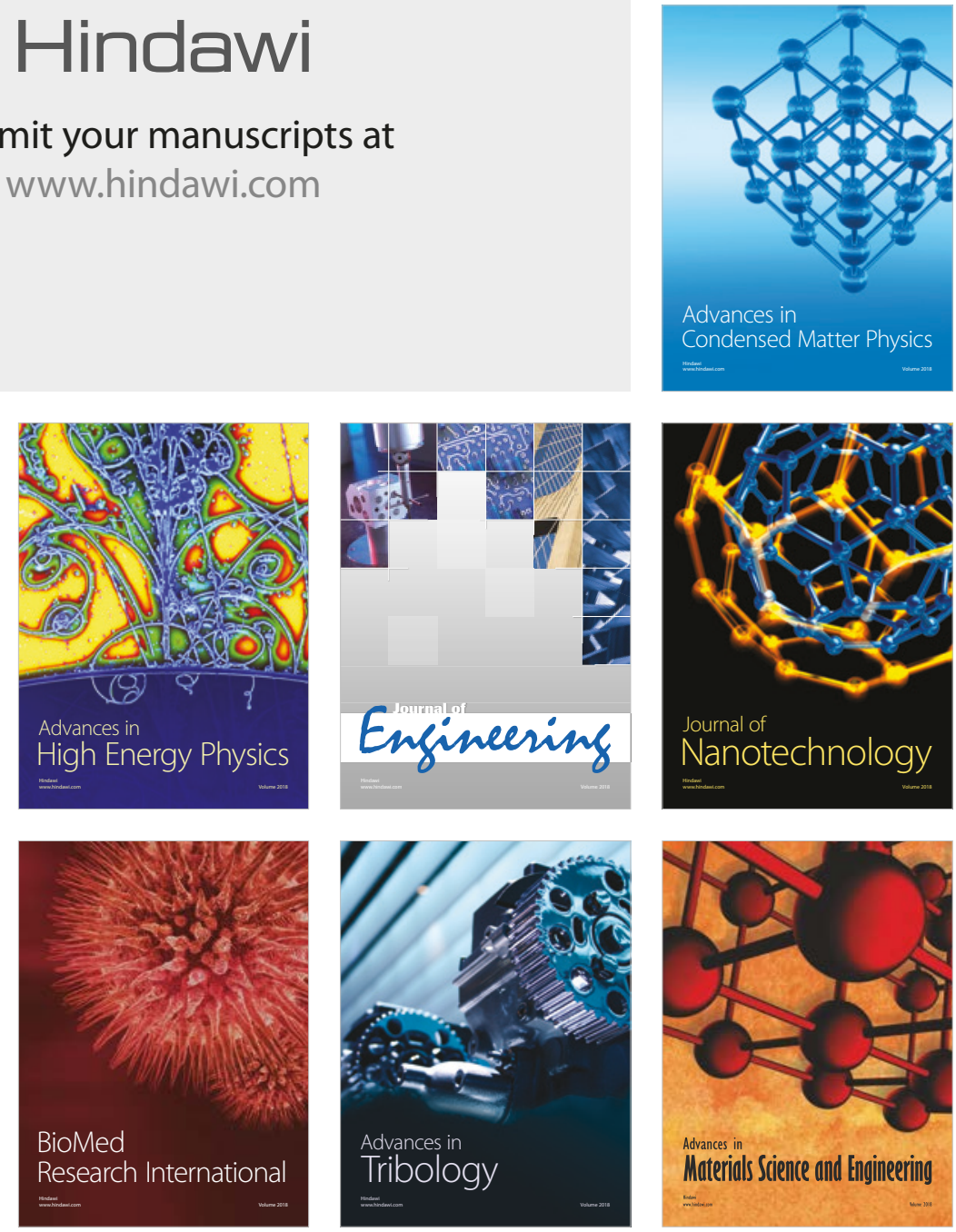